\section{Max Lüscher}

$\mathrm{M}$ an reagiert auf eine Empfindung entweder vernünftig und der Realität angemessen oder unvernünftig, weil man aus Gefühlen einer egozentrischen Befangenheit reagiert.

\section{Gefühl und Vernunft}

Zwei Menschen können, wenn sie gegen eine Tischkante stossen, unterschiedlich reagieren. Zunächst versetzt der Stoss beide in eine Erregung. Jedoch der eine schimpft: „Diese blöde Tischkante geht mir auf die Nerven.“ Die Empfindung des Stosses erzeugt eine Überreizung und damit eine psycho-somatische Belastung. Die Empfindung des Stosses bewirkt bei ihm dadurch ein Gefühl des Widerwillens.

Der andere ist ebenfalls erschrocken und findet: „An dieser Tischkante kann man sich stossen. Ich muss den Tisch anders stellen. “ Diese Haltung ist eine der Realität angemessene und daher eine vernünftige Reaktion. Die Vernunft erzeugt keine psycho-somatische Belastung.

Diese zwei unterschiedlichen Haltungen, die der Realität angemessene Vernunft einerseits oder die egozentrischen Gefühle andererseits, bestimmen, wie man sein Leben gestaltet. Die egozentrische Haltung führt zum Scheitern, zu Konflikten, zu Misserfolgen und zur körperlichen Erkrankung.

Die der Realität angemessene, vernünftige Haltung führt zur Offenheit, zum Verständnis der Situation und der anderer Menschen. Sie ist die Voraussetzung zur psychisch-somatischen Gesundheit und zugleich $\mathrm{zu}$ einer ethischen Haltung gegenüber anderen.

Die zwei gegensätzlichen Haltungen, die vernünftige und die ego-zentrische, bestimmen nicht nur den Alltag, sondern auch den Verlauf des eigenen Lebens. Jeder ist frei, seinem Leben ent-

\title{
Ursache psycho-somatischer Erkrankungen: Die Vermischung von Denken und Fühlen
}

weder einen vernünftigen Sinn zu geben, oder es im sinnlosen Teufelskreis von ego-zentrischen Gefühlen und Konflikten zu vergeuden.

\section{Die gesunde Selbst-Steuerung durch die Vernunft}

Das der Realität angemessene Verständnis erreicht man, wenn man sich bei jeder Verstimmung fragt: "Was habe ich missverstanden, dass ich mich ärgere, dass ich beleidigt bin, dass ich enttäuscht oder unzufrieden bin?" Diese Frage ist auch dann erforderlich, wenn man zwar selbst im Recht ist, aber die Situation des anderen oder der Gesellschaft nicht versteht.

\section{Die Unterscheidung von} Sinnes-Wahrnehmung, SinnesEmpfindung, Gefühl, Auffassung

- Die Wahrnehmung eines fremden Reizes heisst Sinnes-Wahrnehmung, z.B. beim Hören: laut oder leise; beim Sehen: hell oder dunkel, dunkelblau oder orange; beim Tasten: glatt oder rau; beim Schmecken: süss oder sauer etc. Auch Pflanzen und Tiere haben Wahrnehmungen, z.B. für Wärme, für Licht etc.

- Die Wahrnehmung des physiologischen Zustandes heisst Sinnes-Empfindung. Die spontane Sinnes-Empfindung ermöglicht eine Beurteilung, z.B. das ist wärmer oder kälter; lauter oder leiser; das ist fressbar oder unfressbar; heller oder dunkler; diese rote Farbe ist erregend oder dieses dunkle Blau ist beruhigend. Das angelernte Wissen kann dem spontanen Empfinden direkt widersprechen, z.B. bedeutet das Rot der Verkehrsampel Erregung als Gefahr und deshalb Stopp. Hier hingegen gibt man der Erregung von Rot nicht die Bedeutung von Antrieb und Vorwärtsbewegung, sondern von Erregung als Gefahr und deshalb Stopp.

- Das Bewusstsein. Die Wahrnehmung einer Empfindung, bei der man sich selbst (als „ICH“) erfährt, heisst: Gefühl, z.B. „Ich ärgere mich“, „Ich freue mich“. Gefühle und Bewusstsein haben auch höher entwickelte Tiere.

- Die Bewertung des eigenen Gefühls heisst: Auffassung oder Meinung z.B. „Ich bin stolz, gewonnen zu haben.“ "Dunkelblau gefällt mir besser als Orange.“

\section{Zusammenfassung:}

- Die Wahrnehmung von physiologischen Reizen heisst: Sinnes-Empfindung.

- Die Wahrnehmung der Sinnes-Empfindung heisst: Gefühl.

- Die Bewertung des eigenen Gefühls heisst: Auffassung (auch bei höheren Tieren).

\section{Das physiologisch begründete Bewusstsein}

Die Wahrnehmung des physiologischen Zustandes des Hippocampus und des limbischen Systems wird als Gefühl empfunden. Ist der physiologische $\mathrm{Zu}$ stand stimuliert, dann wird er als Erregung empfunden. Ist er sediert, dann wird er als Ruhe empfunden. Ist er kontrahiert, dann wird er als Festigkeit empfunden. Ist er dilatiert, dann wird er als Lösung empfunden.

\section{Das egozentrisch-emotionale Bewusstsein}

Meist wird die Empfindung des physiologischen Zustandes, z.B. der Erregung, mit einer Vorstellung (im Neocortex) gekoppelt, zum Beispiel mit der Vorstellung: Mein Kollege ärgert mich. Zwischen der Vorstellung, „mein Kol- 
lege“ und der physiologischen „Erregung“ besteht eine ständige Rückkoppelung und Wechselwirkung. Weil ich erregt bin, muss ich an diesen Kollegen denken und, weil ich an diesen Kollegen denke, rege ich mich auf. Diese Wechselwirkung schaukelt sich auf und bewirkt eine Verstärkung, die Vernunft wird blockiert. Weil man den anderen für den Verursacher hält und ihm die Schuld gibt, fühlt man sich im Recht und in der egozentrischen Meinung bestärkt.

\section{Die Unvernunft als Krankheit}

Durch die Rückkopplung der egozentrischen Emotionen entstehen stark wirksame Motivationen. Sie können sich zur explosiven Heftigkeit steigern, z.B. bei der unbegründeten Eifersucht (Othello) oder beim Amokläufer und den sog. borderline Reaktionen.

Bei der Unvernunft als Krankheit wird die emotionale Egozentrizität nicht als die eigene Ursache erkannt, sondern die Schuld wird auf eine vorhandene Situation oder eine Beziehung projiziert, auf einen anderen Menschen, auf den Partner, auf das Kind, auf die Kindheit oder auf ein Objekt: „Diese dumme Tischkante.“ Die Rückkopplung der egozentrischen Emotionen steigert sich am häufigsten zu depressiven Haltungen, oft auch zu manischen oder zu zwanghaften und illusionären Zuständen.

Beispiel: Ein junger, freundlich wirkender Patient geriet in diese Rückkopplung. Er arbeitete in einem Grossraumbüro. Plötzlich stand er auf, ging zu seinem ihm wohl gesonnen Chef und sagte: „Ich weiss, dass Sie mich ständig beobachten und denken, dass ich ungenügend bin. Das halte ich nicht mehr aus. Ich kündige.“

Eine Vorstellung, die der Realität nicht angemessen und daher unvernünftig ist, wird immer masslos überbewertet $(++)$ oder masslos abgewertet (--). Sie explodiert zur Schwärmerei bei der Verliebtheit, bei Rockkonzerten, im Fussballstadion oder bei konfessionellen und politischen Massendemonstrationen oder sie äussert sich als Abwertung, als Missachtung und Verachtung oder als aggressive Intoleranz und Negation „Die anderen sind blöd“ oder als Voreingenommenheit „Das ist doch alles Quatsch“.

Tab. 1. Die normativen Selbst-Gefühle zur psycho-somatischen Therapie

\begin{tabular}{|lll|}
\hline $\begin{array}{l}\text { Die normativen } \\
\text { Selbst-Gefühle }\end{array}$ & $\begin{array}{l}\text { Die ego-zentrischen } \\
\text { Gefühle }\end{array}$ & $\begin{array}{l}\text { Das ego-zentrische } \\
\text { Verhalten }\end{array}$ \\
Die Selbst-Achtung & beleidigt sein & trotzig sein \\
Das Selbst-Vertrauen & entmutigt sein & bequem, träge sein \\
Die eigene Freiheit & ängstlich sein & sich unsicher fühlen \\
Die eigene Zufriedenheit & unzufrieden sein & gierig, süchtig sein \\
\hline
\end{tabular}

Durch die ständige Rückkopplung wird der egozentrische Zustand zum gewohnten, aber abnormen Selbst-Gefühl, z.B. man ist ständig beleidigt: „Man wird mir nicht gerecht. Man demütigt mich“ oder man ist gehemmt: „Ich kann es ja doch nicht“ oder ist eingebildet: „So gut wie ich ist keiner“.

Die meisten Menschen verharren in einem dieser un-vernünftigen, egozentrischen Zustände. Sie sind verärgert, aggressiv, verletzend, trotzig oder arrogant, beleidigt, enttäuscht oder sie klagen, weinen und bedauern sich oder sie sind schwärmerisch und unvernünftig leidenschaftlich. Durch die ständige Rückkopplung wird der egozentrische Zustand zum fortwährend bestehenden Bewusstsein und gewohnten, aber abnormen Selbst-Gefühl.

Das Selbst-Gefühl erzeugt die vorherrschende Haltung. Sie beeinflusst das Schicksal und verursacht die psycho-somatische Krankheitsdisposition. Wenn sich die abnormen Selbst-Gefühle fixieren, entsteht der paranoide Wahn z.B. der Minderwertigkeits- oder Grössen-Wahn und die Formen der Angst.

\section{Der emotionale Angelhaken}

Viele können sich trotz ihres Verstandes und trotz ihres besseren Wissens von einer schädlichen Bindung nicht lösen. Sie sagen, der quälende Angelhaken sitzt nicht im Kopf, sondern im Herzen oder im Bauch.

Warum kann man den schädlichen Angelhaken trotz Wissen, trotz Einsicht und Verstand nicht entfernen? Weil die Vorstellung (im Neocortex) an das Gefühl (im limbischen System) gekoppelt ist. Das Gefühl ist das Bedürfnis, nicht allein zu sein oder die Erinnerung an glückliche Augenblicke oder an die sexuelle Befriedigung. Das Wissen und der Verstand können die schäd- liche Rückkopplung und die quälenden Gefühle nicht aufheben.

\section{Die Entkopplung}

Die illusionären Wunsch-Vorstellungen sind der Angelhaken, an dem wir hängen. „Was der andere tut, ist zwar schlecht, aber vielleicht wird er doch noch so, wie ich es mir wünsche.“

Aus der Sackgasse der Rückkopplung gibt es nur den Ausweg über die Vernunft. Vernunft ist die Einsicht in die objektive Wirklichkeit. Die Vernunft respektiert die Wirklichkeit und nimmt sie ernst, ohne Konzessionen zu machen. Sie erfordert den Willen zur Wahrhaftigkeit und verlangt eine unerbittliche Selbstdisziplin. Sie lässt keine Ausreden zu.

Im dem Augenblick, da man sich zur Selbstdisziplin und zur Wahrhaftigkeit entschieden hat, in dem Augenblick hat die Vernunft begriffen, was der Verstand zwar wusste, aber nicht bewirken konnte. Dieser Augenblick ist das Aha-Erlebnis. Man sagt: „Jetzt ist mir ein Licht aufgegangen“ oder „Jetzt ist der Groschen gefallen.“

\section{Die traumatische Fixierung}

Schädliche Rückkopplungen können zu traumatischen Fixierungen führen. Sie verursachen eine charakterliche Entartung. Diese äussert sich in zahllosen Formen eines unangemessenen Verhaltens von der Süchtigkeit bis zur Kriminalität, von der Depression bis zum Suizid.

\section{Die normativen Selbst-Gefühle zur psycho-somatischen Therapie}

Aufgabe der Psychotherapie ist es, die schädlichen, egozentrischen Rückkopplungen zu erkennen und mit dem Willen zur Wahrhaftigkeit zu entkoppeln. Wenn das gelingt, dann treten an- 
stelle der egozentrischen Selbst-Gefühle die vier normalen Selbst-Gefühle: Die Selbst-Achtung, das Selbst-Vertrauen, die eigene Freiheit und die $\mathrm{Zu}$ friedenheit (Tab. 1). Therapien, die das nicht in nützlicher Frist erreichen, reden an der Aufgabe vorbei.

\section{Das der Realität angemessene Bewusstsein}

Werden egozentrisch über- oder unterbewertete Emotionen vermieden, dann kann die Realität angemessen und vernünftig beurteilt werden, dann erlebt man eine harmonische Übereinstimmung mit der Realität. Sie fördert die Kommunikation und die emotionale Resonanz, das vernünftige Denken, das ästhetische Erleben und das praktische Handeln.

Das der Realität adäquate, vernünftige Bewusstsein ist das Ziel der täglichen Selbst-Steuerung, der Erziehung, der Pädagogik, einer vernünftigen Psychotherapie und einer psycho-somatischen Medizin.

\section{Literatur}

Lüscher M: Die beiden Taschenbücher „Der 4Farben Mensch" und "Das Harmoniegesetz in uns" (beide Ullstein Verlag, Berlin) sind im Buchhandel erhältlich.

Die Tabellen "Das ICH als Ursache von Konflikten und Depressionen" sowie das Hörbuch "Der 4-Farben-Mensch" sind nur erhältlich "bei: www.luscher-color.com

\section{Anschrift des Autors:}

Prof. Dr. Max Lüscher

Kreuzbuchrain 14, CH-6006 Luzern

profluscher@paso.ch

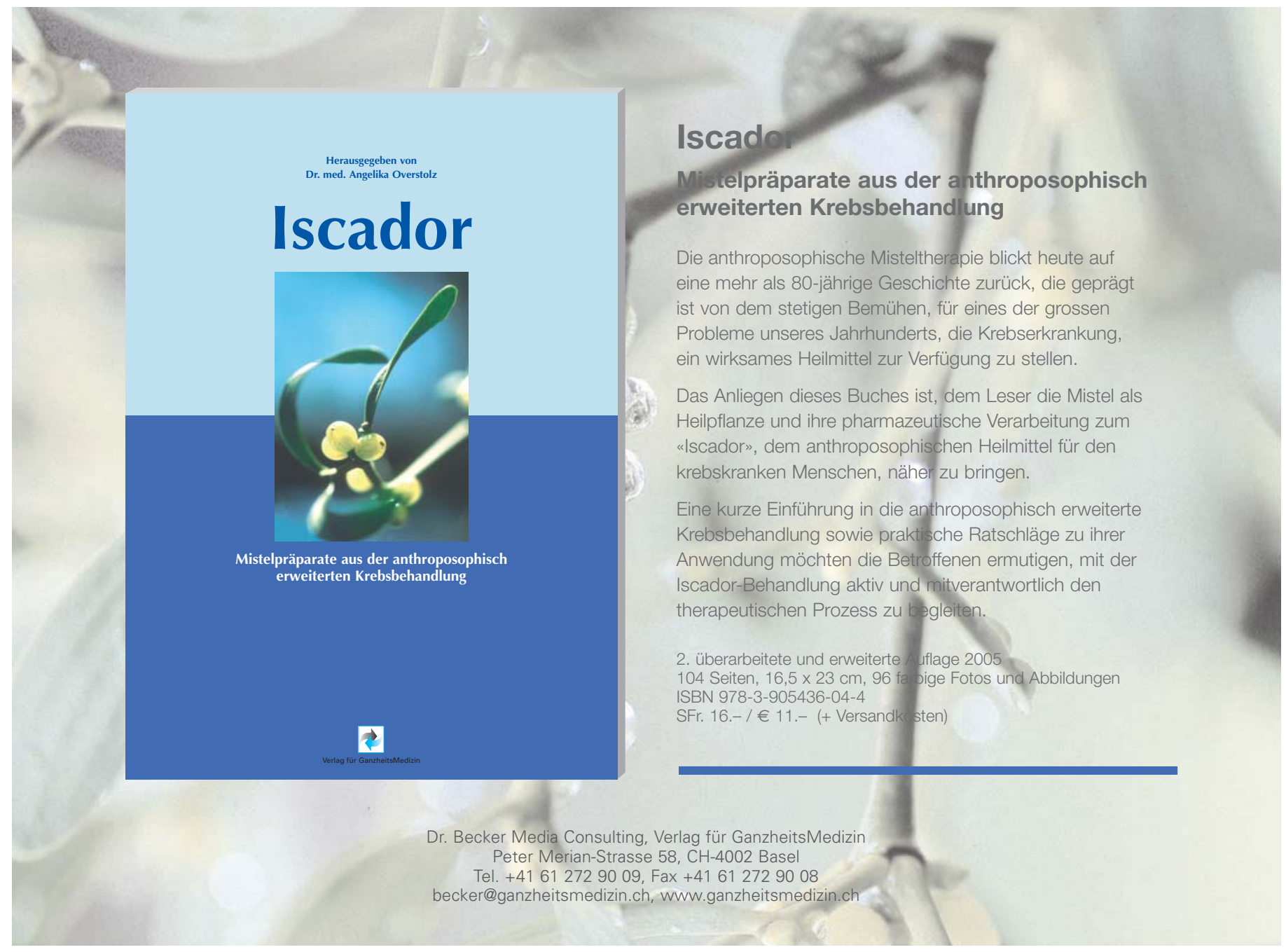

